\title{
EDITORIAL
}

Open Access

\section{Why Materials Theory?}

\author{
Anter El-Azab
}

Correspondence:

aelazab@purdue.edu

School of Materials Engineering,

Purdue University, West Lafayette,

IN 47907, USA
Materials science is an interdisciplinary field with the broad objectives of understanding the structure and properties of materials and the discovery of new materials. In his historical account of this field titled The Coming of Materials Science (Cahn et al. 2003), R. W. Cahn referred to the middle of the past century as the time materials science was born out of metallurgy. Materials science has expanded since then to cover the science of ceramics, polymers, semiconductors, and numerous functional materials. Since the inception of materials science, experiment has been a central theme underlying investigation of the structure and properties of materials while modelling was aimed initially at the interpretation of experimental results. However, with the need to understand increasingly complex materials structures and the connection of materials structure with materials behaviour, advanced theoretical concepts from the fields of physics, chemistry, mechanics, applied mathematics, and statistics were introduced. Thus, the development of rigorous models for materials structure, materials defects, microstructure evolution, and the behaviour of materials became a second thrust of materials science. Over the past three decades, the theory of materials has worked hand in hand with experiments to interpret results and to explore materials behaviour under conditions that at present cannot be probed directly by experiments. Concurrently, materials research began to exploit the rapidly increasing power of computers to solve theoretical models and to generate structural and property related data through simulations. This in turn enabled materials discovery for applications, including batteries (Liu et al. 2015) and structural materials (Schmitz et al. 2011). The availability of advanced simulation tools capable of predicting the structure and behaviour of materials over varying length and time scales and the possibility of integrating such tools into materials design marked the coming of integrated computational materials engineering (ICME) (Integrated Computational Materials Engineering: A Transformational Discipline for Improved Competitiveness and National Security et al. 2008), an approach integrating simulation tools at all relevant scales for the concurrent design of materials, processes, and products. Furthermore, the quest for accelerated materials discovery has ushered in the era of the materials genome (MG), where experiments, computational tools, and big data (Materials Genome Initiative Strategic et al. 2014) are combined to accelerate materials discovery. Advances in computing, data acquisition, and the discovery and design of materials have also led to the application of the principles of informatics to materials (Rajan 2005), whereby information based on the structure and property of materials and their connections are surveyed to enable MGand ICME-type efforts.

At this point in time, it is fair to state that materials research is driven by materials discovery and engineering. In this regard, understanding the structure of materials and

(c) The Author(s). 2017 Open Access This article is distributed under the terms of the Creative Commons Attribution 4.0 International License (http://creativecommons.org/licenses/by/4.0/), which permits unrestricted use, distribution, and reproduction in any medium, provided you give appropriate credit to the original author(s) and the source, provide a link to the Creative Commons license, and indicate if changes were made. 
structure-property connections remain key materials research goals toward which experiments, theoretical modelling, and computations will continue to be used in concert. Moreover, continued advances in materials simulation tools require continued development of theoretical materials models. Furthermore, in this era of data-driven materials science, theory serves as a basis for encoding materials data, as with thermodynamic data of materials, and in generating such data, as in using, say, ab initio or mesoscale models to generate structure and property data at various length and time scales. The theory of materials will thus continue to play a major role in advancing materials science, not only at the level of developing mathematical models describing the ever increasing complexity of materials but also in enabling predictive data-driven materials research. Accordingly, an avenue to promote materials theory will thus be important in shaping future directions in the field of materials science. Recognizing the prominent role of theory, the editor initiated discussions regarding the founding of a theory-focused journal with members of the community around the world approximately 5 years ago. Initial brainstorming sessions with Professor Michael Zaiser of The University of Erlangen-Nuremberg and Dr. James Belak of Lawrence Livermore National Laboratory were particularly useful in clarifying the need for such a journal. Motivated by the excitement associated with the perceived role of materials theory and by the absence of "theory-oriented research" in particular within the existing materials publishing venues, further discussions with the community at large continued before a decision to approach Springer with the idea. The preponderance of scientists contacted shared the same enthusiasm for the idea, and a number of them have been incorporated into the editorial board of the new journal, Materials Theory.

Materials Theory is intended for the publication of original research articles, review articles, letters to the editor, rapid communications, and thematic collections in all areas of theoretical materials science and related computations. The journal has an interdisciplinary scope spanning mechanics, physics, and chemistry of materials, and concerning materials structure, synthesis, design, and performance. Providing demonstrable progress in the conceptual, mathematical, and computational formalisms of materials research is essential for the acceptance of manuscripts for publication in Materials Theory. The decision to make Materials Theory an open access provides authors a venue for rapid dissemination of their research in as much as users everywhere will be able to access the journal articles without financial or institutional barriers.

Competing interests

The author declares that he has no competing interests.

Received: 4 May 2017 Accepted: 4 May 2017

Published online: 25 May 2017

References

R.W. Cahn, The Coming of Materials Science, 2nd Edn. (Pergamon, 2003).

Integrated Computational Materials Engineering: A Transformational Discipline for Improved Competitiveness and National Security, Committee on Integrated Computational Materials Engineering, National Materials Advisory Board, Division on Engineering and Physical Sciences, National Research Council, National Academies Press (2008).

M Liu et al., Spinel compounds as multivalent battery cathodes: a systematic evaluation based on ab initio calculations. Energy Environ. Sci. 8, 964-974 (2015)

Materials Genome Initiative Strategic Plan, National Science and Technology Council, Committee on Technology, Subcommittee on the Materials Genome Initiative (2014).

K Rajan, Materials Informatics. Mater. Today 8, 38-45 (2005)

GJ Schmitz et al., Towards integrative computational materials engineering of steel components, production engineering. Research and Development 5, 373-382 (2011) 